\title{
Martin's Axiom and a Regular Topological Space with Uncountable Net Weight Whose Countable Product is Hereditarily Separable and Hereditarily Lindelöf
}

Krzysztof Ciesielski

West Virginia University, krzysztof.ciesielski@mail.wvu.edu

\section{Digital Commons Citation}

Ciesielski, Krzysztof, "Martin's Axiom and a Regular Topological Space with Uncountable Net Weight Whose Countable Product is Hereditarily Separable and Hereditarily Lindelöf" (1987). Faculty Scholarship. 819.

https://researchrepository.wvu.edu/faculty_publications/819 
The Journal of Symbolic Logic

Volume 52, Number 2, June 1987

\title{
MARTIN'S AXIOM AND A REGULAR TOPOLOGICAL SPACE WITH UNCOUNTABLE NET WEIGHT WHOSE COUNTABLE PRODUCT IS HEREDITARILY SEPARABLE AND HEREDITARILY LINDELÖF
}

\author{
KRZYSZTOF CIESIELSKI ${ }^{1}$
}

In $[1$, p. 51] A. V. Arhangel'skiĩ, in connection with the problems of $L$-spaces and $S$-spaces, examined further the notions of hereditary separability and hereditary Lindelöfness. In particular he considered the following property P: "Every regular topological space has a countable net weight provided its countable product is hereditarily Lindelöf and hereditarily separable." He noticed that the continuum hypothesis implies negation of the property $\mathrm{P}$ and posed a question: "Do Martin's Axiom and the negation of the continuum hypothesis imply P?" The purpose of this paper is to give a negative answer to this question.

The set-theoretical and topological notation that we use is standard and can be found in [6] and [5] respectively.

Throughout the paper we will use the notation $H(X, Y)$ to denote the set of all finite functions from a set $X$ to $Y$.

Theorem. Con $(\mathrm{ZFC}) \rightarrow \mathrm{Con}(\mathrm{ZFC}+\mathrm{MA}+\neg \mathrm{CH}+$ there exists a 0-dimensional Hausdorff space $X$ such that $n w(X)=\mathfrak{c}$ and $n w(Y)=\omega$ for any $Y \in[X]^{<\mathfrak{c}}$.

ProOF. Let $M$ be a model of ZFC satisfying $\mathrm{CH}$ and let $F$ be an $M$-generic filter over the Cohen forcing $\left(H\left(\omega_{2} \times \omega_{2}, 2\right), \supset\right)$. Then $f=\bigcup F$ is a function and $f: \omega_{2}$ $\times \omega_{2} \rightarrow 2$.

In $M[f]$, define the functions $f_{\zeta}: \omega_{2} \rightarrow 2$ by $f_{\zeta}(\eta)=f(\eta, \zeta)\left(\zeta, \eta<\omega_{2}\right)$ and consider $X=\left\{f_{5}: \zeta<\omega_{2}\right\}$ as a topological subspace of $2^{\omega_{2}}$.

For $\varepsilon \in H\left(\omega_{2}, 2\right)$, let $[\varepsilon]$ be the element of the standard basis of $2^{\omega_{2}}$ (i.e. $[\varepsilon]=$ $\left\{g \in 2^{\omega_{2}}: \varepsilon \subset g\right\}$ ). For $\alpha<\omega_{2}$, let

$$
Q_{\alpha}=\left\{\langle A, \varepsilon\rangle: \varepsilon \in H\left(\omega_{2}, 2\right) \text { and } A \subset\left([\varepsilon] \cap\left\{f_{\zeta}: \zeta<\alpha\right\}\right) \text { and }|A|<\omega\right\}
$$

with ordering $\langle A, \varepsilon\rangle \leq\langle B, \delta\rangle$ if and only if $A \supset B$ and $\varepsilon \supset \delta$.

\footnotetext{
Received April 15, 1986.

'The following results are part of the author's Ph.D. thesis written at the University of Warsaw under Professor Pawel Zbierski. I am most grateful to him and Wojciech Guzicki for their kind help. I also wish to thank my colleagues at Bowling Green State University for their hospitality in 1985/86.
}

(C) 1987, Association for Symbolic Logic $0022-4812 / 87 / 5202-0006 / \$ 01.40$ 
It is easy to see that $Q_{\alpha} \in M\left[f\left\lceil\omega_{2} \times \alpha\right]\right.$ for $\alpha<\omega_{2}$. Moreover let $Q$ be a direct product of the forcings $Q_{\alpha}$, i.e.

$$
Q=\operatorname{II}\left\{Q_{\alpha}: \alpha<\omega_{2}\right\}=\left\{h: \operatorname{dom}(h) \in\left[\omega_{2}\right]^{<\omega} \& h(\alpha) \in Q_{\alpha} \text { for every } \alpha \in \operatorname{dom}(h)\right\},
$$

ordered by

$$
h \leq h^{\prime} \text { if and only if } \operatorname{dom}(h) \supset \operatorname{dom}\left(h^{\prime}\right) \text { and } h(\alpha) \leq h^{\prime}(\alpha) \text { for every } \alpha \in \operatorname{dom}\left(h^{\prime}\right) .
$$

Let $G$ be an $M[f]$-generic filter over $Q$.

In $[4, \S 5]$ (compare also [3, Theorems 2 and 4$]$ ) we proved that the forcing $Q$ satisfies the ccc property in the model $M[f]$ and the model $M[f][G]$ satisfies

$$
n w(X)=\mathfrak{c}=\omega_{2} \text { and } n w(Y)=\omega \text { for any } Y \in[X]^{<\mathfrak{c}}
$$

(The second part of (*) follows immediately from the fact that if $Y \subset\left\{f_{\zeta}: \zeta<\alpha\right\}$ for some $\alpha<\omega_{2}$ and $N_{k}=\{f \in Y: f \in A$ for some element $\{\langle\alpha+k,\langle A, \varepsilon\rangle\rangle\} \in G\}$, then the family $\left\{N_{k}: k<\omega\right\}$ forms a countable network for $Y$.) We now give a generic extension of $M[f][G]$ which preserves the property $(*)$ and satisfies Martin's Axiom.

In $M[f][G]$ let $R$ be an iteration with finite supports of ccc forcings of the form $T_{\alpha}=\left\langle\omega_{1}, \leq_{\alpha}\right\rangle$ such that $R \Vdash \mathbf{M A}+\left(\mathrm{c}=\omega_{2}\right)$ (compare for example $[2, \$ 6, \mathrm{pp} .444-$ 451]). Hence $R=H\left(\omega_{2}, \omega_{1}\right)$ and, for $g, g^{\prime} \in R, g \leq g^{\prime}$ if and only if $\operatorname{dom}(g) \supset$ $\operatorname{dom}\left(g^{\prime}\right)$ and $g\left\lceil\alpha \Vdash g(\alpha) \leq_{\alpha} g^{\prime}(\alpha)\right.$ for every $\alpha \in \operatorname{dom}\left(g^{\prime}\right)$.

Let $H$ be an $M[f][G]$-generic filter over $R$. Clearly

$$
M[f][G][H] \vDash M A+\left(c=\omega_{2}\right) .
$$

Moreover for every $Y \subset 2^{\omega_{1}}$ the sentence " $N$ is a network of $Y$ " is absolute; by the ccc property of $R$, for every $Z \in[X]^{\omega_{1}}$ from $M[f][G][H]$ there exists $Y \in[X]^{\omega_{1}}$ from $M[f][G]$ such that $Z \subset Y$. Hence

$$
M[f][G][H] \vDash n w(Y)=\omega \text { for every } Y \in[X]^{<c} .
$$

Note that since $h L(Y) \leq n w(Y)$ we have (in $M[f][G][H]) h L(Y)=\omega$ for every $Y \in[X]^{<c}$. This implies $M[f][G][H] \models h L(X)=\omega(\mathrm{cf}$. [5] or [4]).

To complete the proof it suffices to show that $n w(X)=\mathrm{c}$ holds in $M[f][G][H]$. The original idea of the proof is that, for $M[f][G], \vDash n w(X)=\omega$ (see [4] or [3]).

By way of contradiction assume that there exists a family $\mathscr{F}=\left\{F_{\zeta}: \zeta<\omega_{1}\right\}$ in $M[f][G][H]$ such that

$$
M[f][G][H] \vDash " \mathscr{F} \text { is a network for } X " .
$$

By the regularity of $X$ the family $\left\{\mathrm{cl}_{X} F_{\zeta}: \zeta<\omega_{1}\right\}$ is also a network for $X$, so we can assume that the sets $F_{5}$ are closed in $X$. Hence, by hereditary Lindelöfness of $X$, there exists in $M[f][G][H]$ a sequence $\mathscr{E}=\left\langle\varepsilon_{n}^{\zeta} \in H\left(\omega_{2}, 2\right): \zeta\left\langle\omega_{1} \& n<\omega\right\rangle\right.$ such that

$$
X \backslash F_{\zeta}=X \cap \bigcup\left\{\left[\varepsilon_{n}^{\zeta}\right]: n<\omega\right\} \text { for all } \zeta<\omega_{1} .
$$

Let $R_{\alpha}$ be an iteration of length $\alpha$ of the forcings $T_{\beta}$ for $\beta<\alpha$ (i.e. $R_{\alpha}=\{g \uparrow \alpha$ : $g \in R\}$ ). Then $H_{\alpha}=R_{\alpha} \cap H$ is an $M[f][G]$-generic filter over $R_{\alpha}$. 
Since the forcing $R$ is $\operatorname{ccc}$ and $|\mathscr{E}| \leq \omega_{1}$, there exists $\alpha<\omega_{2}$ such that $\mathscr{E} \in$ $M[f][G]\left[H_{\alpha}\right]$.

For $\beta<\omega_{1}$ put $\bar{Q}^{\beta}=\prod\left\{Q_{\gamma}: \beta \leq \gamma<\omega_{2}\right\}, G^{\beta}=G \cap \bar{Q}^{\beta}$ and $G_{\beta}=G \cap \Pi\left\{Q_{\gamma}\right.$ : $\gamma<\beta\}$. We know that $Q$ is $\operatorname{ccc}$ in $M[f]$ and $\left|R_{\alpha}\right| \leq \omega_{1}$; thus there exists $\beta<\omega_{2}$ such that $R_{\alpha} \in M[f]\left[G_{B}\right]$.

Hence, by the product lemma,

$$
M[f][G]\left[H_{\alpha}\right]=M[f]\left[G_{\beta}\right]\left[G^{\beta}\right]\left[H_{\alpha}\right]=M[f]\left[G_{\beta}\right]\left[H_{\alpha}\right]\left[G^{\beta}\right],
$$

i.e.

$$
\mathscr{E} \in M[f]\left[G_{\beta}\right]\left[H_{\alpha}\right]\left[G^{\beta}\right] .
$$

But in $M[f]\left[G_{\beta}\right]$ we have $\bar{Q}^{\beta} \Vdash R_{\alpha}$ is ccc; consequently $\bar{Q}^{\beta} \times R_{\alpha}$ is ccc and $R_{\alpha} \Vdash \bar{Q}^{\beta}$ is ccc. Hence in $M[f]\left[G_{\beta}\right]\left[H_{\alpha}\right]$, the forcing $\bar{Q}^{\beta}$ is ccc and $|\mathscr{E}| \leq \omega_{1}$.

Therefore there exists $\gamma<\omega_{2}$ such that $\beta<\gamma$ and

$$
\mathscr{E} \in M[f]\left[G_{\beta}\right]\left[H_{\alpha}\right]\left[G_{\gamma}^{\beta}\right]=M[f]\left[G_{\gamma}\right]\left[H_{\alpha}\right]
$$

where $G_{\gamma}^{\beta}=G^{\beta} \cap \Pi\left[\left\{Q_{\delta}: \beta \leq \delta<\gamma\right\}\right.$. Moreover $\prod\left\{Q_{\delta}: \delta<\gamma\right\} \in M\left[f \mid \omega_{2} \times \gamma\right]$, i.e.

$$
M[f]\left[G_{\gamma}\right]=M\left[f \uparrow \omega_{2} \times \gamma\right]\left[G_{\gamma}\right]\left[f \uparrow \omega_{2} \times\left(\omega_{2} \backslash \gamma\right)\right] .
$$

Hence there exists $\delta<\omega_{2}, \delta>\gamma$, such that

$$
R_{\alpha} \in M\left[f\left\lceil\omega_{2} \times \gamma\right]\left[G_{\gamma}\right]\left[f \uparrow \omega_{2} \times(\delta \backslash \gamma)\right]=M\left[f \uparrow \omega_{2} \times \delta\right]\left[G_{\gamma}\right] .\right.
$$

In particular we have

$$
\mathscr{E} \in M\left[f \lceil \omega _ { 2 } \times \delta ] [ G _ { \gamma } ] [ H _ { \alpha } ] \left[f\left\lceil\omega_{2} \times\left(\omega_{2} \backslash \delta\right)\right] .\right.\right.
$$

Let $N=M\left[f\left\lceil\omega_{2} \times \delta\right]\left[G_{\gamma}\right]\left[H_{\alpha}\right], a \in \omega_{2} \backslash \bigcup\left\{\operatorname{dom}\left(\varepsilon_{n}^{\zeta}\right): \zeta<\omega_{1} \& n<\omega\right\}\right.$ and $f_{\delta}(a)=i$, and put

$$
\phi \equiv "\left(\forall \zeta<\omega_{1}\right)\left(f_{\delta} \notin F_{\zeta} \text { or } F_{\zeta} \not \subset[\{\langle a, i\rangle\}]\right) " .
$$

We next prove

$$
N\left[f \uparrow \omega_{2} \times\left(\omega_{2} \backslash \delta\right)\right] \vDash \phi .
$$

Fix $\zeta<\omega_{1}$ and assume that $f_{\delta} \in F_{\zeta}$. Then there exists $s \in H\left(\omega_{2} \times\left(\omega_{2} \backslash \delta\right), 2\right)$ such that

$$
N \vDash s \Vdash\left(f_{\delta} \in F_{\zeta}=X \backslash \bigcup\left\{\left[\varepsilon_{n}^{\zeta}\right]: n<\omega\right\}\right) .
$$

But $\left\langle\varepsilon_{n}^{\zeta}: n\langle\omega\rangle \in N\right.$, i.e. the last statement is equivalent to

$$
X \cap[\varepsilon] \subset X \backslash \bigcup\left\{\left[\varepsilon_{n}^{\zeta}\right]=F_{\zeta},\right.
$$

where $\varepsilon=\{\langle\xi, i\rangle:\langle\xi, \delta, i\rangle \in s\}$. Hence, for $\varepsilon^{\prime}=\varepsilon \uparrow \bigcup\left\{\operatorname{dom}\left(\varepsilon_{n}^{\zeta}\right): n<\omega\right\}, X \cap\left[\varepsilon^{\prime}\right]$ $\subset F_{\zeta}$. Put $\varepsilon^{\prime \prime}=\varepsilon^{\prime} \cup\{\langle a, 1-i\rangle\}$. Then

$$
\varnothing \neq\left[\varepsilon^{\prime \prime}\right] \cap X \subset\left[\varepsilon^{\prime}\right] \cap X \subset F_{\zeta} \text { and }\left[\varepsilon^{\prime \prime}\right] \cap X \cap[\{\langle a, i\rangle\}]=\varnothing ;
$$

i.e. $F_{\zeta} \not \subset[\{\langle a, i\rangle\}]$. This completes the proof of $(* *)$.

To finish the proof of the theorem first observe that the sentence $\phi$ is absolute, hence $M[f][G][H]=$ " $\phi$ " (i.e. $M[f][G][H] \models \mathscr{F}$ is not a network for $X$ ). This contradicts our assumption. 
COROLlaRY. $\operatorname{Con}(\mathrm{ZFC}) \rightarrow \operatorname{Con}(\mathrm{ZFC}+\mathrm{MA}+\neg \mathrm{CH}+$ there exists a regular space $X$ such that $n w(X)=\mathrm{c}$ but $\left.h L\left(X^{\omega}\right)=h d\left(X^{\omega}\right)=\omega\right)$.

Proof. It is enough to show that the condition " $n w(Y)=\omega$ for every $Y \in[X]^{\omega_{1}}$ " implies $h L\left(X^{\omega}\right)=h d\left(X^{\omega}\right)=\omega$. So let us assume that $n w(Y)=\omega$ for every $Y \in$ $[X]^{\omega_{1}}$. Then for every $n<\omega$ and $Y \in[X]^{\omega_{1}}$ we have $h L\left(Y^{n}\right) h d\left(Y^{n}\right) \leq n w\left(Y^{n}\right)=\omega$. Therefore $h L\left(X^{n}\right)=h d\left(X^{n}\right)=\omega$ and hence (see for example [1,p. 51]) $h L\left(X^{\omega}\right)$ $=h d\left(X^{\omega}\right)=\omega$.

\section{REFERENCES}

[1] A. V. ARHANGEL'SKiı̆, The structure and the classification of topological spaces and cardinal invariants, Uspehi Matematičeskih Nauk, vol. 33 (1978), no. 6 (204), pp. 29-83 (Russian); English translation, Russian Mathematical Surveys, vol. 33 (1978), no. 6, pp. 33-96.

[2] J. P. BurGEss, Forcing, Handhook of mathematical logic (J. Barwise, editor), North-Holland, Amsterdam, 1977, pp. 403-453.

[3] K. CiesielsK1, On the netweight of subspaces, Fundamenta Mathematicae, vol. 117 (1983), pp. $37-46$.

[4] - - The topologies generated by graphs, Proceedings of the Jadwisin conference 1981, University of Leeds Press, Leeds, 1983, pp. 67-92.

[5] I. JuHÁsz, Cardinal functions in topology - ten years later, Mathematisch Centrum, Amsterdam, 1980.

[6] K. KunEN, Set theory, North-Holland, Amsterdam, 1980,

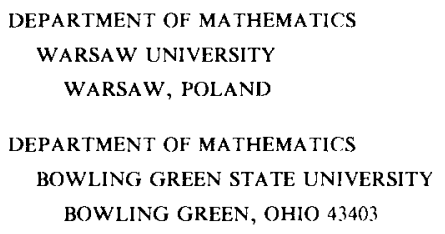

The Warsaw address is the permanent one. 